\section{PTH-075 LONG-TERM OUTCOMES AFTER ENDOSCOPIC RESECTION OF LARGE COLORECTAL SUPERFICIAL NEOPLASTIC LESIONS IN AN EXPERT CENTRE}

Andrew Emmanuel, Shraddha Gulati, Anil Ghosh, Christo Lapa, Margaret Burt, Bu Haji, Amyn Haji. King's College Hospital, London, UK

\subsection{6/gutjnl-2018-BSGAbstracts.96}

Introduction There are very few western data describing the long term outcomes of endoscopic resection of large colorectal superficial neoplastic lesions (CSNL) beyond 12-18 months. The best available data suggests most recurrence occurs early whereas late recurrence (after 3-6 months) is rare and not a significant problem. Given the widespread and increasing use of advanced endoscopic resection techniques, it is important to define the long term outcomes beyond the first year of surveillance.

Methods We analysed endoscopic resections (ER) of large $(\geq 2 \mathrm{~cm})$ CSNL performed at a tertiary referral centre. Surveillance is conducted according to a standardised protocol involving magnification chromoendoscopy and NBI performed at 3-6 months and 12 months, followed by standard adenoma surveillance again at 3 years. Recurrence rates at $<6$ months, 12-23 months, 24-35 months and $\geq 36$ months follow up were calculated. Recurrence rates at each interval were only calculated for patients in whom no prior recurrence had been detected at an earlier interval. $\chi^{2}$ test was used to determine factors significantly associated with early recurrence $(<12$ months) and late recurrence ( $\geq 12$ months).

Results 588 ER of large CSNL were performed (mean size $52.5 \mathrm{~mm}$ ). After relevant exclusions, 425 patients had undergone at least the first surveillance endoscopy, of which 259 had undergone 12 month follow up, 90 had undergone $\geq 24$ months follow up and $51 \geq 36$ months follow up. Recurrence at $<6$ months occurred in 34 (8\%). 15/235 (6.4\%) patients had recurrence at 12 month surveillance. $7 / 70$ (10\%) had recurrence from 24-35 months follow up and 4/35 (11.4\%) developed recurrence after $\geq 36$ months follow up. Factors significantly associated with recurrence were similar for both early and late recurrence (size $>40 \mathrm{~mm}$, piecemeal resection, EMR vs ESD, prior heavy manipulation, use of APC). However, of patients followed up $\geq 36$ months ( $n=51$ ), 93\% had successful ER (including treatment of recurrence) and have remained free from recurrence at last follow up.

Conclusions These data demonstrate that recurrence after apparent successful ER of large CSNL continues to occur after 2 and even 3 years of follow up, despite subsequent normal initial surveillance. Although recurrence at 12 months was similar to large published series, significant proportions of patients continue to develop recurrence with longer term follow up. Nevertheless, ER in an expert centre is safe and highly effective for the vast majority.

\section{PTH-076 EFFECT OF INCREASING EXPERIENCE ON OUTCOMES OF COLORECTAL ENDOSCOPIC RESECTION IN A LARGE WESTERN SERIES}

Andrew Emmanuel, Shraddha Gulati, Margaret Burt, Bu Hayee, Amyn Haji. King's College Hospital, London, UK

\subsection{6/gutjnl-2018-BSGAbstracts.97}

Introduction Despite decades of experience in Japan, endoscopic mucosal resection (EMR) and particularly endoscopic submucosal dissection (ESD) for large colorectal lesions are relatively new techniques in western practice. It is well recognised in surgery that for new techniques, the relevance of early published studies can change rapidly as experience grows. Given the increasing use and experience in expert centres of endoscopic resection (ER), the relevance of initial published results of endoscopic resection in western centres may change. We determined the effect of increasing experience on resection strategies and outcomes in a large series of colorectal ERs at a tertiary referral centre.

Methods We analysed all endoscopic resections of large $(\geq 20 \mathrm{~mm})$ colorectal lesions performed at a tertiary referral unit between January 2011 and December 2017. ERs were arbitrarily divided into 2 time periods (Period 1: January 2011 - December 2014 and Period 2: January 2015 December 2017). Comparisons of outcomes of ER were made between these 2 time periods, including use of ESD, en bloc resection, successful initial ER, complications, perforations and recurrence detected at the first surveillance colonoscopy (3-6 months).

Results Endoscopic resection was performed for 588 colorectal lesions (mean size $52.5 \mathrm{~mm}$ ) during the study period (Period 1 $\mathrm{n}=264$, Period $2 \mathrm{n}=324)$ of which $578(98.3 \%)$ were initially successful. Comparing Periods 1 and 2, there were no differences in overall complications $(6.1 \%$ vs $4.6 \%, \mathrm{p}=0.44)$ or perforations $(3.0 \%$ vs $3.1 \%, \mathrm{p}=0.97)$ and initial success of ER was similar $(98.1 \%$ vs $98.5 \%, \mathrm{p}=0.74)$. However, the use of ESD was far more common in Period $2(p<0.001)$ and en bloc resection was achieved more frequently in Period 2 $(p=0.002)$. Recurrence detected at the first surveillance colonoscopy was significantly less frequent during Period 2 (5.6\% vs $10.8 \%, \mathrm{p}=0.05)$.

Conclusion These data demonstrate the evolving nature of endoscopic resection practice in a western expert centre. The findings confirm that expert performed EMR is safe and effective, but refining and developing resection strategies with increasing experience, incorporating ESD more frequently, results in higher en bloc resection rates and probably improved initial adenoma clearance as demonstrated by less frequent recurrence at first surveillance. This underscores the importance of appropriate referral to expert centres that have developed the necessary expertise.

\section{Endoscopy Video}

\section{OWE-007 SALINE-IMMERSION THERAPEUTIC ENDOSCOPY (SITE) FOR ENDOSCOPIC SUBMUCOSAL DISSECTION (ESD) OF A LARGE RECTAL LESION}

Alberto Murino*, Andrea Telese, Nikolaos Lazaridis, Nikolaos Koukias, Edward I Despott. Royal Free Unit for Endoscopy, The Royal Free Hospital and University College London (UCL) Institute for Liver and Digestive Health, Hampstead, London, London, UK

\subsection{6/gutjnl-2018-BSGAbstracts.98}

Introduction Since its first description in 2012, underwater endoscopic mucosal resection has become a well-recognised alternative approach to standard submucosal-injection facilitated endoscopic mucosal resection. In 2017, our group first described Saline-immersion therapeutic endoscopy (SITE) as an 'evolution of the underwater technique' with several potential advantages. ${ }^{1}$ To date, only limited data concerning the use of immersion methods for endoscopic submucosal dissection 